\title{
Wavelet Analysis for Multiresolution Tissue Characterization in Intracoronary Optical Images
}

\author{
Maysa M G Macedo ${ }^{1}$, Pedro FG Nicz², Carlos M Campos ${ }^{2}$, Pedro A Lemos ${ }^{2}$, Marco A Gutierrez ${ }^{2}$ \\ ${ }^{1}$ IBM Research, Sao Paulo, Brazil \\ ${ }^{2}$ Heart Institute, University of Sao Paulo Medical School, Brazil
}

\begin{abstract}
The rupture of atherosclerotic plaques in coronaries is responsible for most acute coronary events. A new imaging modality called intravascular optical coherence tomography (IOCT) enables a detailed high resolution visualization of micro-structural changes of the in vivo arterial wall and provides an accurate representation of the thickness of a fibrous cap and a useful tool to assess the vulnerability in lipid-rich plaques. This article describes a fully automated method for tissue characterization in IOCT human coronary images. In this study the continous wavelet transform is used to analyze plaque tissue in IOCT frames in polar coordinates, enabling a quantification of fibrous cap thickness. In-vivo human coronary images were used in experimental tests for detecting fibrous cap automatically, where the results were compared with manual segmentations from the same expert in two different instants separated by 2 months.
\end{abstract}

\section{Introduction}

Coronary artery disease (CAD) is one of the leading causes of mortality world-wide [1]. CAD is intrinsically related to atherosclerosis, in which an inflammed vessel wall forms an atherosclerotic plaque. The rupture of this plaques is responsible for most acute coronary events. Thin-cap fibroatheroma (TCFA) is characterized by a necrotic core with an overlying thin fibrous cap $(\leq 65 \mu \mathrm{m})$ [2], which has been used as indicator of plaque vulnerability [3]. Yabushita et al [4] concluded Intravascular Optical Coherence Tomography (IOCT) can accurately characterize plaque components with a high degree of sensitivity and specificity, identifying morphological features of coronary plaques at risk of rupture. Recent technological advances in intravascular imaging technology such as optical coherence tomography (IOCT) provide an accurate representation of the thickness of the fibrous cap and is a useful tool to assess the vulnerability of lipid-rich plaques [5].
Fibrous tissue has a texture in IOCT images with highest backscattering of all image areas. However changes may occur in brightness according to the position of the catheter at the time of frame acquisition. Methods based only on texture analysis [6] and intensity of the pixels may be adversely affected by changes in light intensity caused by the catheter movement. Thus, frequency domain-based methods can overcome these image intensity problems. In addition, another challenge in the analysis is that the fibrous cap does not usually have fixed size in images with inflamed vessel walls, thus requiring a method that analyze plaques with various resolutions. The continuous wavelet transform can analyze the fibrous cap at several resolutions, differing positively from other methods of spacefrequency analysis.

\section{Method}

There are several methods of time or distance frequency analysis, including the Short-time Fourier transform (STFT), Wigner-Ville distribution (WVD), ChoiWilliams distribution (CWD) and Continuous wavelets transform (CWT). But the objective is the same: analyze spectrum for each time or distance interval. Macedo et al [7] used STFT to quantify fibrous cap, however that method is dependent on window size and the result does not reflect tissue heterogeneities. In this study we used CWT to do the same task, but with a multiresolution approach.

\subsection{Data}

Images were acquired using a Fourier-Domain OCT (FD-OCT) system (C7-XR - OCT Intravascular Imaging System, St. Jude Medical, St. Paul, Minnesota) at the Heart Institute, University of Sao Paulo Medical School (INCOR-HC FMUSP), Brazil. The study protocol was approved by the institutional review board (CAPPesq) under register no. 0243/08. Pullbacks speeds were between 20 and $25 \mathrm{~mm} / \mathrm{sec}$ over a distance of $54.0 \mathrm{~mm}$, totaling 271 and 541 frames respectively. The $2 \mathrm{D}$ input images to the 
proposed method were in DICOM format with dimensions $1024 \times 1024$ pixels and spatial resolution of $10 \times 10 \mu \mathrm{m}$ in Cartesian coordinates.

In total, 54 2D images from 22 patients were used for testing. Manually segmented reference images were used to assess the automatic quantification. An independent observer, blinded to the automatic fiber delineation, analyzed the same set of IOCT frames and manually delineating the fibrotic cap. The same delineations were repeated after 2 months by the same observer to provide a measure of intraoperator variability.

The manual segmentation protocol was divided in two stages: lipid pool delimitation and fibrous cap delineation. However, the lipid pool delimitation was only performed during the first segmentation. For the second time, the Expert used the same lipid pool delimitation to define the fibrous cap. The manual delineations were performed using ImageJ software.

\subsection{Multiresolution analysis}

The term wavelet refers to an oscillatory window function of frequency $f_{o}$ whose width is finite and compactly supported. The Continuous Wavelet Transform (CWT) is expressed as:

$$
C W T(\sigma, a)=\frac{1}{\sqrt{(a)}} \int_{-\infty}^{\infty} x(s) \cdot \Psi *\left\{\frac{s-\sigma}{a}\right\} d t
$$

The function $\Psi(s)$, the mother Wavelet, is selected depending on the type of the signal. The factor $\frac{1}{\sqrt{(} a)}$ normalizes the energy of the signal. $a$ is the scale of the wavelet and $\tau$ represents the mother wavelet shifting along the signal $x(s)$. The result of this transformation is a scalogram (scale $\times$ time or space) as presented in Figure 3 a which represents the percentage of energy for each coefficient.

\subsubsection{Wavelets for IOCT}

Figure 1 shows the developed method for application of the CWT to the identify fibrous cap. For this study, we used the intensity profile for each column of a 2D IOCT in polar coordinates as input signal for the CWT. Note that this polar image is centered in the lumen centroid, the lumen border was extracted with a method described in [8]. Each column of this image (A-line) is a intensity profile as depicted in Figure $2 \mathrm{~b}$.

For each degree in the polar image, the CWT computes the matching between the input signal and the mother wavelet at several scales. Our method selects a specific scale finding the higher magnitude in the scalogram. The scalogram is binarized using $3 \%$ of the maximum magnitude as threshold, where the idea is to adquire the region where there is more wavelet matching. It is used an area

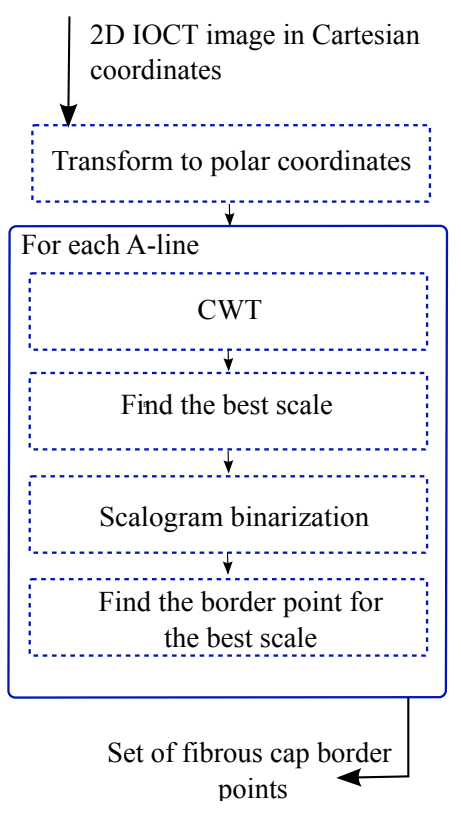

Figure 1. Scheme of the proposed method to segment fibrous cap.
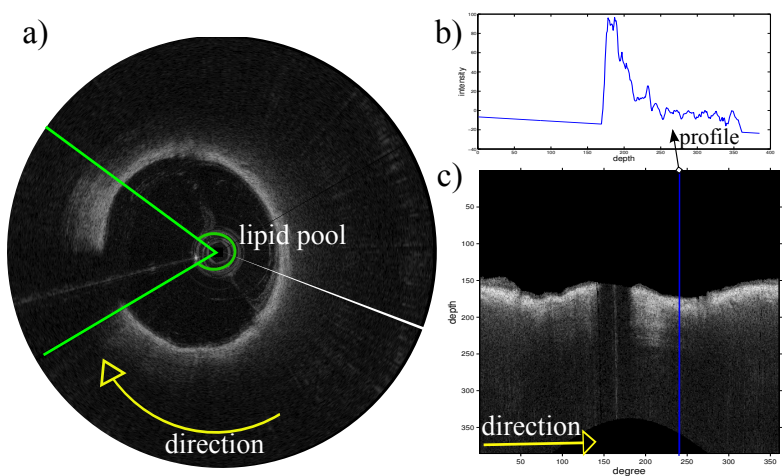

Figure 2. Process to get the input signal. a) a 2D IOCT image in Cartesian coordinates. The obtuse triangle indicates the lipid pool region b) A-line with its intensity profile c) the IOCT image in polar coordinates centred on lumen centroid, the arrow indicates the direction of the transformation compared with the Cartesian one. The vertical blue line in the polar image shows a single A-line scan, whose profile is plotted above.

constraint in the binary image (Figure $3 \mathrm{c}$ ) to detach only the maximum area. Using the chosen scale, the method selects the border in the binary region. In Figures $3 a-b$ the cross indicates the point selected as fibrous cap border, it shows the binarization step and the border points identified in the whole image.

The mother wavelet used is the Haar. This choice was defined by the signal profile, the fiber cap has a high intensity and then with the lipid pool the intensity drops 

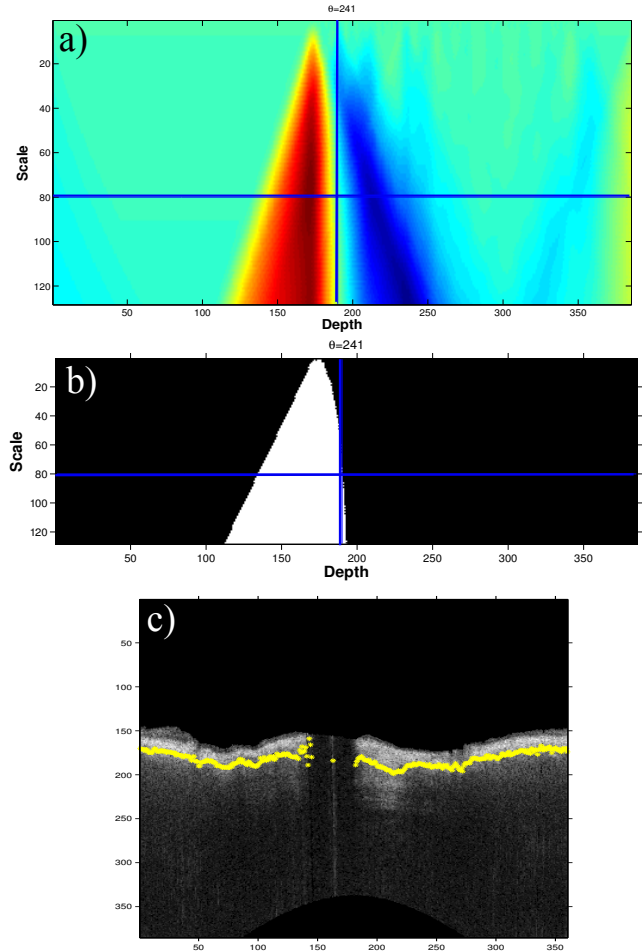

Figure 3. The process to identify the cap border point. a) Scalogram for $\theta=241$ where the top indicates magnitudes for small scales and high frequencies. b) The binarization of the scalogram, where the cross indicates the point selected as fiber cap border. c) The border points for all $\theta$ values.

abruptly. Preprocessing was performed in the input signal using an approach to eliminate the signal linear trend and apply a moving average.

\section{Results}

The evaluation metrics were computed considering only the fibrous cap above the lipid pool region. The metrics are the result of comparison with manual and automatic fibrous cap delineation such as: Dice metric, Accuracy (ACC), Sensitivity (S), Specificity(SPE), and finally Mean Absolute Difference of fibrous cap area (MADA).

Tests were carried out on a total of 54 images with atherosclerotic plaque obtained during 22 acquisitions. The fibrous cap area was obtained without any user interaction. The evaluation of the fibrous tissue segmentation method was based on manual segmentation by an Expert. The mean difference between the automated and the manual fibrous cap area was found to be $0.15 \mathrm{~mm}^{2}$. Considering the overlap of both results, the automated method achieved $80 \%, 78.0 \%$ and $99.5 \%$ for Dice metric, sensitivity and specificity, respectively. Table 3 shows all eval- uation metrics of the proposed automated method and the comparison between both manual segmentations. Figure 4 shows the results of automated fibrotic tissue identification compared with manual segmentation. And Figure 5 illustrates the thickness along the A-lines of the same images in Figure 4 comparing the automated method with the two manual procedures.

Table 1. Results of automated delineation of fibrous cap in IOCT images compared with manual delineations

\begin{tabular}{lccr}
\hline \hline Metric & Auto x ExT1 & Auto x ExT2 & ExT1 x ExT2 \\
\hline Dice $(\%)$ & $80.3 \pm 7.0$ & $80.0 \pm 7.2$ & $87.9 \pm 5.0$ \\
MADA $\left(\mathrm{mm}^{2}\right)$ & $0.15 \pm 0.17$ & $0.22 \pm 0.3$ & $0.13 \pm 0.2$ \\
S $\%)$ & $78.3 \pm 9.5$ & $78.1 \pm 12.4$ & $84.2 \pm 9.9$ \\
SPE $(\%)$ & $99.8 \pm 0.16$ & $99.8 \pm 0.18$ & $99.9 \pm 0.07$ \\
ACC $(\%)$ & $99.6 \pm 0.34$ & $99.5 \pm 0.4$ & $99.7 \pm 0.3$ \\
\hline \hline
\end{tabular}

mean \pm standard deviation

Auto- Automated method, ExT1- Expert in time 1, ExtT2- Expert in time 2
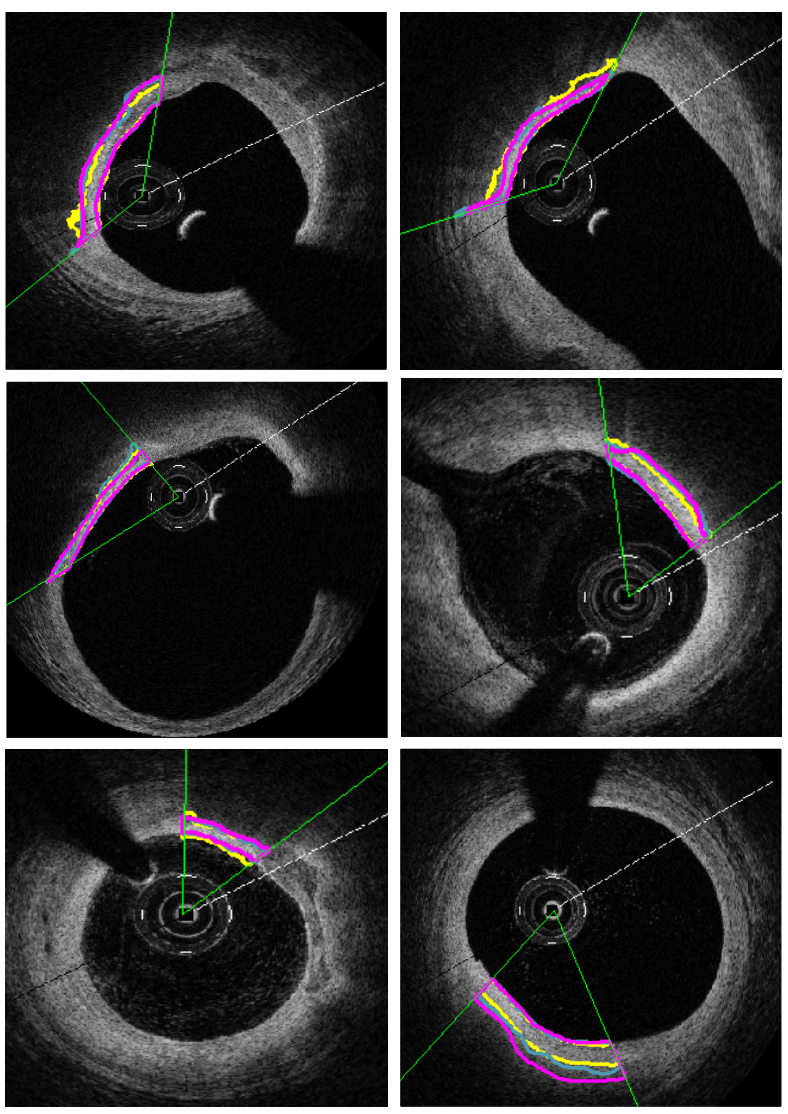

Figure 4. Results of the proposed method for segmentation of fibrous cap. Automated results are in yellow, and both references are in magenta and dark green. The lipid pool was defined only manually by the Expert as two lines in green. 

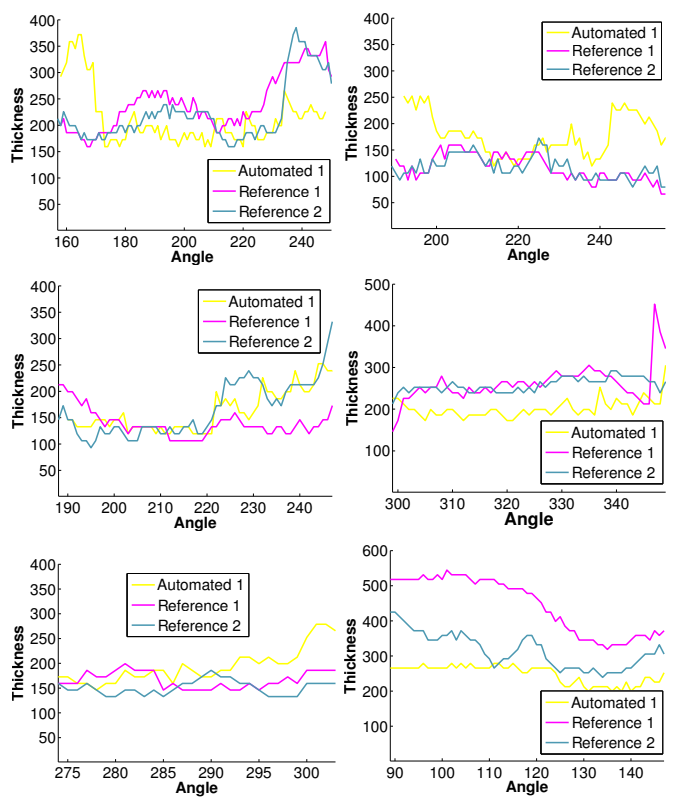

Figure 5. Relation between thickness and each angle in the fibrous cap. Each graph is related to segmentations on Figure 4, respectively.

\section{Conclusions}

The variability of manual segmentations is an important well discussed issue in Radu et al. [9], where they mentioned the high variability between the manual segmentations and they compared the metrics with automated segmentations, which mostly performs the same way. As this proposed method is a deterministic algorithm, it provides the same result for the same image every time.

In terms of comparison, Macedo et al [7] developed a solution to quantify fibrous cap using STFT. However, this solution is not multiresolution, so the results depends on the difference between the window function size used in Fourier transform and the thickness of the fibrous cap. That method, achieved $76 \%$ of Dice metric, which is lower than this current solution. Ughi et al. [6] developed an automated tissue characterization based on texture analysis, attenuation coefficient and pixel classification using Random Forest, reaching an accuracy of $89.5 \%$ for fibrotic tissue, which is lower than our method (99.6\%). Athanasiou et al. [10] developed an automated method based on segmentation and classification using K-means and achieved $87 \%$ and $0.09 \mathrm{~mm}^{2}$ for sensitivity and MADA respectively, better than our solution.

An advantage of the proposed wavelet method over works $[6,10]$ is that it does not require a training stage, and we believe our results can be improved with a parameters tuning for scalogram binarization. Moreover, the multiresolution aspect of wavelets can be also used for lipid and calcium plaque characterization. We presented in this study a non-complex method for measuring fibrous cap thickness that achieved comparable results with two different manual segmentations.

\section{References}

[1] Organization WH. Key Facts. Cardiovascular diseases fact sheet sep 2016;

[2] Sakakura K, Nakano M, Otsuka F, Ladich E, Kolodgie FD, Virmani R. Pathophysiology of atherosclerosis plaque progression. Heart Lung and Circulation 2013;22(6):399-411. ISSN 14439506.

[3] Burke AP, Farb A, Malcom GT, L Iang Y, Mialek JS, Virmani R. Coronary Risk Factors and Plaque Morphology in Men with Coronary Disease who Died Suddenly. The New England Journal of Medicine 1997;336:1276-82.

[4] Yabushita H. Characterization of human atherosclerosis by optical coherence tomography. Circulation sep 2002; 106(13):1640-1645. ISSN 00097322.

[5] Kume T, Akasaka T, Kawamoto T, Okura H, Watanabe N, Toyota E, Neishi Y, Sukmawan R, Sadahira Y, Yoshida K. Measurement of the thickness of the fibrous cap by optical coherence tomography. American heart journal oct 2006; 152(4):755.e1-4. ISSN 1097-6744.

[6] Ughi GJ, Adriaenssens T, Sinnaeve P, Desmet W, D'hooge J. Automated tissue characterization of in vivo atherosclerotic plaques by intravascular optical coherence tomography images. Biomedical Optics Express July 2013; 4(7):1014-30. ISSN 2156-7085.

[7] Macedo MMG, Nicz P, Campos C, Takimura CK, Lemos PA, Gutierrez MA. Spatial-frequency approach to fibrous tissue classification in intracoronary optical images. In Computing in Cardiology, volume 43. 2016; 477-480.

[8] Macedo MMG, Guimarães WVN, Galon MZ, Takimura CK, Lemos PA, Gutierrez MA. A bifurcation identifier for IV-OCT using orthogonal least squares and supervised machine learning. Computerized Medical Imaging and Graphics 2015;46:237-48. ISSN 1879-0771.

[9] Radu MD, Yamaji K, García-García HM, Zaugg S, Taniwaki M, Koskinas KC, Serruys PW, Windecker S, Dijkstra J, Räber L. Variability in the measurement of minimum fibrous cap thickness and reproducibility of fibroatheroma classification by optical coherence tomography using manual versus semi-automatic assessment. EuroIntervention 2016;12(8):e987-e997. ISSN 1969-6213.

[10] Athanasiou LS, Bourantas CV, Rigas G, Sakellarios AI, Exarchos TP, Siogkas PK, Ricciardi A, Naka KK, Papafaklis MI, Michalis LK, Prati F, Fotiadis DI. Methodology for fully automated segmentation and plaque characterization in intracoronary optical coherence tomography images. Journal of Biomedical Optics 2014;19(2):026009.

Address for correspondence:

Maysa M G Macedo

Rua Tutoia, 1157 - Sao Paulo, Brazil, 04007-900

mmacedo@br.ibm.com 